\title{
DÉFINIR LE PARADOXE : DE LA LOGIQUE A LA LINGUISTIQUE
}

\begin{abstract}
A bstract. Wolowska Katarzyna, Définir le paradoxe : de la logique à la linguistique [Between logics and linguistics -- towards a definition of paradox]. Studia Romanica Posnaniensia, Adam Mickiewicz. University Press, Poznań, vol. XXXII : 2005, pp. 97-112. ISBN 83-232-1465-4, ISSN 0137-2475.
\end{abstract}

The linguistic paradox - considered in a perspective of the discourse analysis - appears to be a mechanism rooted in two relations: the oppositional one (contradiction or contrariness) and the junction obtaining between the same components. The interpretation of the paradox can also be implied (or, sometimes, even forced) by using terms or markers directly stating its presence (paradoxical, paradoxically, it seems paradoxical that, etc.); still, each componential opposition can be neutralized in the discourse, which makes contradiction and contrariness canceled on the level of semantic interpretation to have later the paradoxical sequence integrated into the "doxical perspective".

\section{INTRODUCTION}

Loin de constituer un phénomène purement linguistique, le paradoxe apparaît comme un de ces problèmes-hybrides qui acceptent, et même nécessitent, une approche pluridisciplinaire pour être compris et expliqués de façon passablement exhaustive. Vu que différents domaines de recherche (philosophie, logique, rhétorique, linguistique) connaissent le paradoxe et l'envisagent en fonction de leurs propres objets d'étude, les définitions de ce phénomène sont nombreuses et parfois assez divergentes les unes par rapport aux autres. Pourtant, ôté les éléments spécifiques marquant leur appartenance aux domaines où elles ont été créées, toutes les définitions présentent en général des points communs qui suffisent pour établir une définition à peu près univoque du paradoxe.

Cet article se veut une sorte de synthèse des définitions que l'on trouve dans divers dictionnaires ou encyclopédies (plus ou moins spécialisés) à l'entrée paradoxe (ou paradoxisme') et qui mériteraient d'être confrontées en quelque sorte aux

' La distinction entre les deux termes, introduite dans les ouvrages consacrés à la rhétorique, me paraît inutile. Telle est aussi la conviction de G. Molinié (1992:240) et de R. Landheer (1996:91). 
définitions élaborées au sein des théories linguistiques. Plus précisément, il s'agit ici d'établir une définition "générale » ou, si l'on veut, "prototypique " du paradoxe à partir de ses différentes formulations, en passant par la logique, la philosophie et la rhétorique (sans oublier bien évidemment la conception «courante » du paradoxe), mais en privilégiant les caractéristiques propres au paradoxe de langue. Une telle définition me semble en effet constituer un point de départ nécessaire, même si souvent implicite, pour les approches sémantiques du paradoxe, dont je ne ferai ici que signaler quelques principales directions (cf. Conclusion).

Considérons les définitions suivantes ${ }^{2}$ :

PARADOXE (paradoce, 1485 ; gr. paradoxos " contraire à l'opinion commune ") 1. Opinion [argument ou proposition] qui va à l'encontre de l'opinion communément admise [(doxa), de la vraisemblance]. 2. Etre, chose, fait [extraordinaire, incompréhensible] qui heurte [la raison] le bon sens [la logique]. 3. Log. Se dit d'une proposition qui est à la fois vraie et fausse (Le Petit Robert 1979 ; entre crochets, les mots ajoutés dans la version électronique du Grand Robert).

PARADOXE fait qui paraît défier la logique parce qu'il présente en lui-même des aspects contradictoires (Grand Larousse de la langue française, Paris 1976).

PARADOXE (du gr. para, contre, et doxa, opinion) - jugement contraire à l'opinion communc. - Le Paradoxe sur le comédien, dialogue en prose de Diderot, insiste sur la nécessité de l'insensibilité pour un interprète désireux de toucher le public. Les paradoxes philosophiques les plus célèbres dans l'Antiquité sont ceux qui ont été développés par Zénon d'Elée pour prouver l'impossibilité du mouvement ; tel est l'argurnent d'Achille : Achille ne peut, théoriquement, rattraper la tortue qui a sur lui une avance, si petite qu'elle soit. Car, pour la rattraper, il devra d'abord arriver au point où celle-ci se trouvait quand il a commencé à courir, puis au point où, pendant ce temps, est arrivée la tortuc, et ainsi de suite à l'infini (Dictionnaire de la philosophie 1964).

PARADOXE opinion contraire à l'opinion commune ; affirmation qui, au premier abord, paraît choquante ou absurde, mais qui, à la réflexion, est conforme à la réalité. PARADOXISME alliance de mots antithétiques et qui paraissent logiquement incompatibles, mais mis en œuvre avec une telle habileté au sein d'une même proposition que, tout en se combattant, ils frappent l'intelligence et dégagent à seconde vue un sens merveilleusement vrai, souvent profond et toujours énergique (Dictionnaire de poétique et de rhétorique 1961).

PARADOXISME un artifice de langage par lequel des idées et des mots, ordinairement opposés et contradictoires entre eux, se trouvent rapprochés et combinés de manière que, tout en semblant se combattre et s'exclure réciproquement, ils frappent

${ }^{2}$ Pour ne pas augmenter excessivement le nombre de ces définitions, je me limite à celles que j'ai trouvé dans les dictionnaires et les encyclopédies français et polonais. Les définitions citécs (ainsi que les explications étymologiques du mot 'paradoxe', cf. section 5) suffisent, à mon avis, pour établir une définition «prototypique » du paradoxe. 
l'intelligence par le plus étonnant accord, et produisent le sens le plus vrai, comme le plus profond et le plus énergique (P. Fontanier, Les Figures du Discours 1968 : 137).

PARADOXE, en Philosophie, c'est une proposition absurde en apparence, à cause qu'elle est contraire aux opinions reçues, \& qui néanmoins est vraie au fond, ou du moins

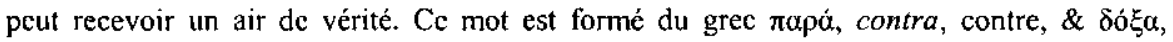
opinion (Encyclopédie 1765).

PARADOXISME, figure de pensée par combinaison, qui consiste à réunir, sur le même sujet des attributs qui, au premicr coup d'œil, paroissent inconciliables \& contradictoircs (Encyclopédie Méthodique 1784).

PARADOKS 1. «pogląd, twierdzenie zaskakująco sprzeczne $z$ ugruntowanymi przekonaniami, często ujęte w formę blyskotliwego aforyzmu» 2. $\log$. «rozumowanie o pozornie oczywistej prawdziwości jego clementów, ale wskutek zawartego w nim blędu prowadzace do wniosków jawnie sprzecznych ze sobą" (fr. paradoxe, z gr. parádoksos 'nieoczekjwany, niewiarygodny') (Komputerowy stownik języka polskiego 1997).

PARADOKS (gr. paradoksos = coś niezgodnego z mniemaniem lub oczekiwaniem) 1. W logice i metodologii: a) terminologiczny równoznacznik antynomii (para zdań, z których każde zasługuje na uznanie, choć zarazem są to zdania między sobą sprzeczne) b) rozumowanie, zachowujące pozory poprawności, w konsekwencjach jednak prowadzące do twierdzeń zaskakujących i (w świetle doświadczenia lub dotychczasowej wicdzy) niemożliwych lub trudnych do przyjęcia. 2 . Twierdzenie sprzeczne $z$ obiegową opinią i nie znajdujące potwierdzenia w aktualnym stanie wiedzy (Mala Encyklopedia filozofii 1996).

PARADOKS (< gr. parádoksos = niespodziewany, dziwny $)$ - efektowne i zaskakujące swoją treścią sformulowanie, zawierające myśl skłóconą z powszechnie żywionymi przekonaniami, sprzeczną wewnętrznie, która jednak przynosi nieoczekiwaną prawdę - filoz.oficzną, moralna, psychologiczną, poetycką, etc. Mechanizm paradoksu opiera się na dwóch operacjach: zestawieniu calości znaczeniowych maksymalnie kontrastowych i ustaleniu między nimi stosunku wzajemnego zawierania się (inkluzji) (Slownik terminów literackich 2000).

PARADOKS (od. lac. paradoxus, od gr. parádoxos, nieoczekiwany, dziwny) 1. sąd, pogląd sprzcczny z ogólnie przyjętymi przekonaniami, wysoce nieprawdopodobny, niezgodny $z$ mocno ugruntowanymi przewidywaniami. 2. pogląd, sąd, wypowiedziany i podtrzymywany bez przekonania, dla zaimponowania błyskotliwościa, dowcipem, oryginalnością (Stownik wyrazów obcych 1964).

PARADOKS podane $w$ blyskotliwej, oryginalnej formie twierdzenie a. rozumowanie sprzeczne (czasem tylko pozornie) z tym, co jest ogólnie uznane za prawdẹ; rozumowanie, w którym (pozornic) poprawnc założenia i wnioskowania prowadzą do sprzeczności i fałszu (gr. paradoksos 'nieoczekiwany; nieprawdopodobny; zadziwiający') (Stownik wyrazów obcych i zwrotów obcojęzycznych 1967).

Ce qui saute aux yeux tout de suite, c'est que les perspectives dans lesquelles est habituellement envisagé le paradoxe s'entre-croisent dans les définitions citées 
sans trop respecter la distinction entre les dictionnaires de langue et les dictionnaires spécialisés. Il semble ainsi important de les préciser, en traitant d'abord les éléments les plus spécifiques et en passant par la définition «courante » du paradoxe pour en arriver aux points communs susceptibles de rendre compte, quelque sommairement que ce soit, de la nature du paradoxe. Evidemment, une telle démarche (analyse des définitions, élimination successive des traits marqués, synthèse des éléments réitérés) ne saurait aboutir à une définition "pure » du paradoxe. Bien au contraire, étant donné que les définitions de dictionnaire ne sont pas tout à fait objectives (mais que signifie "être objectif " dans le cas d'une définition lexicale ?), il est clair que la formulation de la définition "prototypique » du paradoxe à laquelle je veux aboutir ne constitue qu'un artefact, une synthèse des intuitions verbalisées dans les dictionnaires et non pas celle de données scientifiques précises.

\section{PERSPECTIVE LOGIQUE}

La première perspective qui s'esquisse à travers les définitions citées, c'est la perspective logique qui enferme le problème du paradoxe dans le champ restreint des catégories de la vérité. L'omniprésence de cette optique est bien significative :

(1) Log. Se dit d'unc proposition qui est à la fois vraie et fausse (Petit Robert 1979).

(2) Fait qui paraît défier la logique parce qu'il présente en lui-même des aspects contradictoires (Grand Larousse de lo langue française, Paris 1976).

(3) un artifice de langage par lequel des idées et des mots, ordinaircment opposés et contradictoires entre cux, se trouvent rapprochés et combinés (Fontanier 1968:137).

(4) proposition absurde en apparence (...) qui néanmoins est vraie au fond, ou du moins peut recevoir un air de vérité (Encyclopédie 1765).

(5) Log. rozumowanie o pozornie oczywistej prawdziwości jego elementów, ale wskutek zawartego w nim błędu prowadzące do wniosków jawnie sprzec znych ze soba (Komputerowy słownik języka polskiego 1997).

(6) W logice i metodologii: terminologičny równoznacznik antynomii (para zdań, z których każde zasługuje na uznanie, choć zarazem są to adania między soba sprzeczne) (Mata encyklopedia filozofii 1996).

(7) sformulowanic, zawierajace myși (...) sprzeczna wewnetrznie (Stownik terminów literackich 2000).

(8) twierdzenic albo rozumowanic sprzeczne (czasem tylko pozomie) $\underline{z}$ tym , co jest ogólnie uznane za prawdę; rozunnowanic, w którym (pozornie) poprawne założenia i wnioskowania prowadza do sprzeczności i falszu (Stownik wyrazów obcych i zwrotów obcojezycznych $1967)^{3}$.

Les expressions soulignées, relatives aux valeurs de vérité, relevent du domaine de la logique pour laquelle le paradoxe a toujours été une sorte de facteur de

${ }^{3}$ Ici et dans les citations suivantes, c'est moi qui souligne (K.W.). 
contrôle, susceptible de mettre en cause les projets formels les plus cohérents. Et il ne s'agit pas là uniquement du paradoxe de langue, que la logique peut ne pas prendre en compte en considérant le langage naturel comme inconsistant (cf. Tarski 1933, dont les idées ont été exposées par Godart-Wendling 1996), mais du mécanisme du cercle vicieux représenté traditionnellement par le célèbre paradoxe du menteur ( « je mens, mais s'il est vrai que je mens, alors je dis la vérité, et par conséquent je mens... »). Comme ce mécanisme est aussi possible à engendrer dans les systèmes logico-mathématiques, il n'est pas étonnant que les logiciens se sont toujours efforcés de le résoudre (cf. les théories de Russell et Whitehead 1910/1927, de Martin 1967, ou de Kripke 1975, ainsi que la solution «temporelle » proposée par Godart-Wendling 1996).

Des définitions si peu spécialisées que celles citées ci-dessus, il résulte que la logique identifie le paradoxe à la contradiction (« relation entre deux termes, deux propositions qui affirment et nient le même élément de connaissance 》, Petit Robert $)^{4}$ : la proposition 'A est vrai et A n'est pas vrai' sera dite paradoxale, puisqu'elle comporte une contradiction interne. Cette explication semble un peu simpliste, étant donné que les efforts des logiciens se sont toujours dirigés vers la solution du paradoxe du menteur (c'est-à-dire vers l'annulation de la contradiction) et que la logique lui attribue parfois la valeur neutre « ni vrai ni faux » qui n'est pas contradictoire, mais qui néanmoins met en cause le grand théorème de la logique binaire, à savoir le principe du tiers exclu. ${ }^{5}$ Pourtant, la conception explicitée dans les définitions de dictionnaire n'est pas erronée dans la mesure où elle met l'accent sur (1) le critère de la vérité sur lequel se fonde toute analyse logique bien qu'il ne soit pas forcément pertinent pour le paradoxe de langue, et (2) sur la co-présence, dans une proposition paradoxale, de deux éléments à valeurs logiques opposées.

\section{PERSPECTIVE PHILOSOPHIQUE}

Là où l'on parle de paradoxe, on pense souvent à la philosophie, ne serait-ce que grâce aux célèbres apories de Zénon d'Elée (l'argument d'Achille, l'argument de la flèche ${ }^{6}$, etc.). Le concept du paradoxe est en effet issu de la dialectique anti-

${ }^{4}$ La contradiction constitue une des relations qui unissent les éléments au sein du carré logique d'Aristote : «L'opposition que j'appelle de contradiction est celle d'une affirmation exprimant un sujet pris universellement à une négation exprimant le même sujet non pris universellement " (Aristote, Organon, éd. 1994 : 90., cité par Bertrand $2000: 108$ ). Mais le langage humain ne se laisse pas déposer " dans un moule logique préétabli » (lbid. : 111), même si Greimas (1966) et ses successeurs ont essayé d'enfermer le sens élémentaire dans un "carré sémiotique » qui est une transposition du carré logique d'Aristote.

${ }^{5}$ Qui énonce que la proposition peut être soit vraje soil fausse, une troisième solution étant impossible.

'La flèche, ayant parcouru la moitié de la distance la séparant de la cible, doit cncorc parcourir la moitié de la distance restante, et ainsi de suite. Elle n'atteint donc jamais la cible. Donc elle ne bouge 
que, il « servait à démontrer l'erreur d'une théorie en montrant que les conséquences de ses principes conduisent à des antinomies » (Rastier 1996:118) et il a été utilisé aussi par les philosophes, dont Zénon est le plus connu. La logique est ici en quelque sorte soumise (comme outil méthodologique) à la philosophie : ce qui caractérise cette dernière, c'est une tendance à raisonner, puisque c'est la vérité qui est l'enjeu des conceptions philosophiques.

(1) Les paradoxes philosophiques les plus célèbres dans l'Antiquité sont ceux qui ont été développés par Żénon d'Elée pour prouver l'impossibilité du mouvement ; tel est l'argument d'Achille : Achille ne peut, théoriquement, rattraper la tortue qui a sur lui une avance, si petite qu'elle soit. Car, pour la rattraper, il devra d'abord arriver au point où celle-ci se trouvait quand il a commencé à courir, puis au point où, pendant ce temps, est arrivée la tortue, et ainsi de suite à l'infini (Dictionnaire de la philosophie 1964).

(2) en Philosophie, c'est une proposition absurde en apparence, à cause qu'elle est contraire aux opinions reçues (Encyclopédie 1765).

(3) efektowne i zaskakujące swoją treścią sformułowanie, zawierające myśl sklóconą z powszechnie żywionymi przekonaniami, sprzeczną wewnętrznie, która jednak przynosi nieoczekiwaną prawdę - filozoficzna, moralna, psychologiczna, poetycka, etc. (Slownik terminów literackich 2000).

(4) rozumowanie, zachowujące pozory poprawności, w konsekwencjach jednak prowadzące do twierdzeń zaskakujących $i(w$ świctle doświadczenia lub dotychczasowej wiedzy) niemożliwych lub trudnych do przyjęcia (Mala Encyklopedia filozofii 1996).

(5) rozumowanie, w którym (pozornic) poprawne załozenia i wnioskowania prowadzą do sprzeczności i fatszu (Stownik wyrazów obcych i zwrotów obcojęzycznych 1967).

"Philosopher " signifie couramment «raisonner» et, dans un sens un peu péjoratif, "raisonner d'une manière savante, compliquée, oiseuse " (Petit Robert 1979). Ce qui caractérise les paradoxes philosophiques (apories), c'est justement cette nature compliquée du raisonnement qui, en plus, s'écarte des opinions reçues (en écoutant Zénon, « Diogène ne trouva rien de mieux que de marcher, prouvant par le fait l'existence du mouvement », Dictionnaire de la philosophie $1964: 220$ ). Dans sa préface aux Paradoxa stoicorum, Cicéron dit à propos de telles propositions : « Comme elles étonnent et vont à l'encontre de l'opinion courante, ils [stoïciens] les appellent eux-mêmes des paradoxes ", (cf. Van der Poel 1996 : 214). En général, la philosophie considère donc comme paradoxal un jugement apparemment juste, mais dont les conclusions sont contraires à l'« opinion commune ».

pas (Ch. Biet et al., Littérature, Techniques littéraires, coll. « Littérature », Magnard 1989: 159). Cf. aussi ces vers du Cimetière marin de Paul Valéry:

Zénon! Cruel Zénon! Zénon d'Elće!

M'as-tu percé de cette flèche ailée

Qui vibre, vole, et qui ne vole pas!

Le son m'enfante et la flèche me tue!

Ah! le soleil... Quelle ombre de tortue

Pour l'âme, Achille immobile à grands pas! 


\section{PERSPECTIVE RHÉTORIQUE}

Si, dans la philosophie et dans la logique, le paradoxe n'est pas considéré comme un fait de langue par excellence, la rhétorique l'envisage justement de ce côté-là : c'est dans la langue et par la langue qu'on crée des paradoxes afin de les utiliser en vue d'un certain effet pragmatique (influence sur le destinataire). Dans les dictionnaires rhétoriques, le paradoxe compte parmi les figures qui, selon la définition de Dumarsais, constituent des « tours de mots et de pensées qui animent ou ornent le discours $"$.

(1) figure de penséc par combinaison, qui consiste à réunir, sur le même sujet des attributs qui, au premier coup d'œil, paroissent inconciliables \& contradictoires (Encyclopédie méthodique 1784).

(2) alliance de mots antithétiques et qui paraissent logiquement incompatibles, (...) [qui] tout en se combattant, frappent l'intelligence et dégagent (...) un sens merveilleusement vrai (Dictionnaire de poétique et de rhétorique 1961).

(3) un artifice de langage par lequel des idées et des mots, ordinairement opposés et contradictoires entre eux, se trouvent rapprochés et combinés (...), tout en semblant se combattre et s'exclure réciproquement, ils frappent l'intelligence par le plus étonnant accord, et produisent le sens le plus vrai (Fontanier 1968 : 137).

(4) efektowne i zaskakujace swoja treścia sformulowanie, zawierające myśl skłóconą z powszcchnie żywionymi przckonaniami (...), która jednak przynosi nicoczekiwaną prawdę. (...) Mechanizm paradoksu opiera siç na dwóch operacjach: zestawieniu całości znaczeniowych maksymalnie kontrastowych i ustaleniu między nimi stosunku wzajemncgo zawjerania się (inkluzji) (Stownik terminów literackich 2000).

(5) pogląd, sąd, wypowiedziany i podtrzymywany bez przekonania, dla zaimponowania bly: skotliwościa, dowcipem, oryginalnościa (Stownik wyrazów obcych 1964).

(6) podane $w$ blyskotliwej, oryginalnej formic twierdzenic a. rozumowanie sprzeczne (czasem tylko pozornie) z tym, co jest ogólnie uznane za prawdę (Stownik wyrazów obcych izwrotów obcojezycznych 1967).

Le paradoxe, envisagé dans la perspective rhétorique, apparaît ainsi comme une combinaison d'éléments de langue (mots ou idées explicitées au moyen de mots, attributs du même sujet, unités significatives, etc.) dont le rapport est normalement celui d'incompatibilité, voire de contradiction, mais qui, rapprochés dans un énoncé, cachent un autre sens, plus vrai et plus profond. Le paradoxe semble donc n'être qu'un moyen, certes un peu pervers mais communicationnellement efficace, de présenter une idée qui paraît bizarre, mais qui, après avoir attiré l'attention du destinataire, s'impose à celui-ci comme tout à fait « doxale ».

En ce qui concerne plus spécifiquement le mécanisme du paradoxe, il est basé sur le rapport d'opposition (qu'on appelle tantôt contradiction, tantôt antithèse, tantôt incompatibilité) entre les éléments rapprochés en discours, ce rapprochement consistant généralement à qualifier un même sujet par des termes qui s'excluent mutuellement ou à établir entre des éléments contraires un rapport d'inclusion 
réciproque. D'autres possibilités d'un tel rapprochement des contraires existent évidemment, mais chacune des définitions citées n'envisage normalement qu'une seule, ou bien - dans la plupart des cas - ne rend compte d'aucune. Ce qu'il est néanmoins nécessaire de prendre en compte, c'est le fait que les auteurs de dictionnaires rattachent souvent à la notion de paradoxe d'autres figures rhétoriques (soit pour leur mécanisme proche de celui du paradoxe, soit pour leurs effets discursifs semblables). Ainsi, parmi les éléments de ce que Douay-Soublin (1996) appelle le « cortège » du paradoxe, on trouvera :

I. antanaclase : « figure de Rhétorique, qui consiste à répéter un mot dans une signification différente \& quelquefois douteuse, comme, laissez les morts enterrer leurs morts " (Abbé Mallet, Encyclopédie 1751: 490, vol. 1) ; « figure de Diction par consonnance physique, qui réunit dans la même phrase des mots de différentes significations, mais matériellement composés des mêmes sons ; comme convenir (être convenable) \& convenir (avouer), voler (s'élever en l'air avec des ailes) \& voler (dérober) " (Beauzée 1782: 192193, tome I) ; « figure dans laquelle le mot répété change de sens, ex. C'est au coeur [milieu] de la société qu'on manque le plus de cceur [charité] » (Morier 1961:111).

II. antithèse : « figure de Rhétorique, qui consiste à opposer des pensées les unes aux autres, pour leur donner plus de jour » (Abbé Mallet 1751:517, vol. 1); « figure de pensée par combinaison, qui, dans la même période ou dans la même tirade, met en opposition des choses contraires, soit par le fond des pensées, soit par le tour de l'expression " (Beauzée 1782 : 203-204, tome I) ; « figure par laquelle on établit un contraste entre deux idées, afin que l'une mette l'autre en évidence» (Morier $1961: 114$ ).

III. contraire : "en Rhétorique, sont les choses opposées les unes aux autres 》 (Abbé Mallet, Encyclopédie, 1754, vol. IV : 121).

IV. opposition : "terme de Rhétorique, c'est une figure de rhétorique, par laquelle l'on joint deux choses qui en apparence sont incompatibles, comme quand Horace parle d'une folle sagesse, \& qu'Anacréon dit que l'amour est une aimable folie. Cette figure semble nier ce qu'elle établit, \& se contredire dans ses termes, est cependant très-élégante ; elle réveille plus que toute autre l'attention et l'admiration des lecteurs, et donne de la grâce au discours, quand elle n'est point recherchée \& qu'elle est placée à propos " (De Jaucourt, Encyclopédie $1765: 513$, vol. XI).

V. oxymore (oxymoron) : "c'est le nom grec donné par les Rhéteurs à la figure que nous appellons opposition" (De Jaucourt $1765: 728$, vol. XI.) ; " mot grec, que quelques rhéteurs ont gardé pour désigner une figure de pensée, que je nomme Paradoxisme " (Beauzée $1784: 744$, tome II) ; "sorte d'antithèse dans laquelle on rapproche deux mots contradictoires, l'un paraissant exclure logiquement l'autre " (Morier 1961: 828) ; " (gr. oksýmoron, od oksýs $=$ ostry + morós $=$ głupi, niedorzeczny) - związek frazeologiczny obejmujący dwa opozycyjne znaczeniowo wyrazy, najczęściej rzeczownik i określający go epitet, rzeczownik i czasownik lub czasownik i przysłówek; w związu takim dochodzi do metaforycznego przekształcenia znaczeń obu słów, dającego efekt paradoksu. Prowokująca nielogiczność oksymoronu sprzyja wydobyciu sensów skomplikowanych, odpowiadających 
dialektycznej złożoności stanów rzeczy, przeżyć czy myśli») (Stownik terminów literackich 2000).

VI. paradiastole : «Figure de pensée par combinaison, qui consiste à distinguer l'une de l'autre des idées analogues \& approchantes, afin de les déterminer d'une manière précise, \& de prévenir la confusion qui pourroit occasionner leur ressemblance. (...) ex. Il y a des gens dont on doit dire, non que leur vie soit oisive, mais qu'ils la passent dans des occupations oiseuses" (Beauzée 1782 : 749-750, tome II).

Le raprochement entre les figures susmentionnées (il y en a d'autres encore) et le paradoxe est caractéristique non seulement des dictionnaires rhétoriques, mais aussi des travaux scientifiques du domaine de la linguistique. Ainsi, par exemple, M. Tuțescu, en proposant le concept d'échelle du paradoxisme, postule qu'on considère «l'amphidoxe, la paradiastole, l'antithèse, l'antiphrase, l'ironie, l'oxymore et la proposition auto-contradictoire comme des aspects de la gradualité paradoxale » $(1996: 83)$.

Dans les définitions du paradoxe liées à la thétorique, il faut compter aussi tout ce qui a trait à la fonction pragmatique de l'emploi d'un énoncé paradoxal. Ainsi, ce dernier « frappe l'intelligence », il est « spectaculaire et surprenant », "brillant et original » et le locuteur l'emploie pour montrer de l'esprit, pour mieux attirer l'attention de son destinataire. La rhétorique étant un " art de bien parler ", une «technique de la mise en œuvre des moyens d'expression du langage " (Petit Robert 1979), le paradoxe apparaît comme un de ses procédés les plus «forts », puisqu'il est basé sur une contradiction (antiphrase, incompatibilité...) sémantique et c'est par par ce biais qu'« il ramène le lecteur à une vérité inattendue » (Riffaterre $\left.1996: 149^{7}\right)$.

\section{PERSPECTIVE LEXICOGRAPHIQUE : VERS UNE DÉFINITION GÉNÉRALE DU PARADOXE DE LANGUE}

Même si elles comportent des éléments spécifiques relevant des domaines logique, philosophique ou rhétorique, les définitions citées ne sont que des entrées de dictionnaires : elles s'adressent soit à un public vaste, voulant s'informer sur la signification du mot sans entrer dans les détails (dictionnaires de langue), soit à un groupe plus restreint de lecteurs plus exigents, désireux d'approfondir leurs connaissances sur tel ou tel concept (dictionnaires encyclopédiques et spécialisés). ${ }^{8}$. Il

${ }^{7}$ En ce qui concerne l'article cité de Riffaterre, il relève de la poétique (dans la mesure où il traite du paradoxe littéraire), mais la conception du paradoxe qui y est proposée s'appuie sur les recherches linguistiques, plus particulièrement sur la sémantique de l'implicite (le paradoxe comme une transgression par rapport aux présuppositions des mots impliqués dans sa structure).

${ }^{8}$ Cette distinction peut paraître un peu trop simpliste, mais elle est suffisante ici. Pour les détails concernant le classement des dictionnaires au sein de la lexicographie, cf. p. ex. J. et Cl. Dubois 1971, surtout les chapitres I et II (7-33). 
est pourtant évident que même les dictionnaires les plus spécialisés ne présentent pas leurs termes de façon exhaustive, car ce n'est pas là leur fonction ; surtout dans le cas des dictionnaires de langue, il s'agit tout simplement de rendre compte du sens courant du mot en tenant compte des différents contextes dans lesquels il apparaît. Il est intéresant de considérer dans cette optique les définitions du paradoxe, à partir de l'étymologie du mot jusqu'à une synthèse des formulations proposées pour expliciter le concept qu'il désigne.

En ce qui concerne l'étymologie du mot paradoxe, les dictionnaires proposent les explications suivantes :

\section{PARADOXE :}

1. du gr. paradoxos, sur doxa, opinion (Dictionnaire étymologique 1964);

2. paradoce, 1485 ; gr. paradoxos « contraire à l'opinion commune" (Petit Robert 1979);

3. gr. parádoksos = niespodziewany, dziwny (Slownik terminów literackich 2000);

4. z gr. parádoksos 'nieoczekiwany, niewiarygodny' (Komputerowy slownik języka polskiego 1997);

5. od. lac. paradoxus, od gr. parádoxos, nicoczekiwany, dziwny (Slownik wyrazów obcych 1964);

6. du gr. para, contre, et doxa, opinion (Dictionnaire de la philosophie 1964) ;

7. gr. paradoksos 'nieoczekiwany ; nieprawdopodobny ; zadziwiający', gr. pará 'poza (czym) ; (tuż) obok, mimo' (Slownik wyrazów obcych i zwrotów obcojęzycznych 1967) ;

8. gr. paradoksos = coś niezgodnego z mniemaniem lub oczekiwaniem (Mala Encyklopedia filozofii 1996).

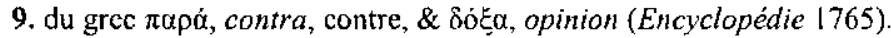

'Paradoxe' est donc un mot composé, créé en grec à partir du mot doxa ('opinion') et du préfixe pará- (traduit de façons assez divergentes comme 'à côté de', 'poza (czym), tuż obok, mimo', 'contre'). Ce dernier indique une certaine différence de la signification du mot composé par rapport à ce qu'exprime le mot radical, différence parfois légère, concernant la relation entre un phénomène et son épiphénomène (cf. 'paralittérature', 'paralangage'), mais parfois allant vers une contradiction partielle (cf. 'paralogisme', 'paramnésie'). Dans le cas du paradoxe, pará- semble introduire cette deuxième nuance de sens, nuance plus « forte », parce que faisant penser à une incompatibilité et non pas à un aspect accessoire, périphérique du mot auquel ce préfixe se rattache.

En ce qui concerne l'idée générale que véhicule le concept de paradoxe dans son acception courante, elle est rendue plus ou moins directement à travers des adjectifs ou des expressions qualificatives tels que "contraire à l'opinion », "qui heurte le bon sens », " inattendu», «bizarre», « invraisemblable », « extraordinaire », «incompréhensible», "surprenant», etc. Deux points centraux de ces définitions s'esquissent ainsi : le premier est lié à la relation d'incompatibilité, voire d'opposition (pouvant aller jusqu'à une contradiction logique), alors que le second concerne une certaine norme sociale, appelée ici doxa, "opinion", mais qui peut 
signifier aussi « attente(s) », " jugement commun », «bon sens », etc. Comme c'est à une définition générale du paradoxe de langue que je voudrais aboutir, il importe de préciser ici non pas la nature de la doxa, mais plutôt le rapport entre celle-ci et les éléments de la langue à base desquels se crée le phénomène en question.

Où placer en effet la relation centrale sur laquelle repose le mécanisme du paradoxe ? Ce terme signifie-t-il au juste l'inadéquation d'un mot, d'un énoncé ou d'une suite d'énoncés à une norme sociale, ou bien le paradoxe consiste-t-il en une co-présence de deux éléments incompatibles, voire contradictoires, reconnus comme tels par rapport à cette norme? Les définitions citées privilégient l'une ou l'autre de ces conceptions selon le point de vue adopté ; il est néanmoins facile de remarquer que, partout où la définition se réfère à l'expression verbale du paradoxe («sformułowanie zawierające myśl sprzeczną wewnętrznie», "alliance de mots antithétiques et qui paraissent logiquement incompatibles ", etc.,), c'est la deuxième conception qui l'emporte. Effectivement, le paradoxe linguistique s'appuie certes sur une certaine norme sociale, codifiée dans le système de la langue ou résultant du contexte discursif, mais son actualisation nécessite la présence des éléments incompatibles sur l'axe syntagmatique du discours; dans le cas inverse, il ne saurait être question que d'un paradoxe situationnel, et celui-ci m'intéresse seulement quand il est explicitement verbalisé. Plus spécifiquement, le critère de «bon sens » permettant de constater l'adéquation ou l'inadéquation d'une séquence (mot, syntagme, énoncé, texte) à une certaine «norme» (sociale, culturelle, idiolectale, mais toujours relative à l'expression linguistique), le paradoxe apparaît comme une relation de jonction établie entre les éléments qui normalement devraient s'exclure.

Quant au nombre d'éléments nécessaires pour pouvoir parler de paradoxe, les définitions considérées indiquent d'une manière plus ou moins explicite que la relation d'incompatibilité qui relie les éléments concernés est une opposition binaire.

Ainsi, dans la logique, il s'agit d'« une proposition à la fois vraie et fausse » ou de deux propositions contradictoires («para zdań») ; dans un dictionnaire de langue, il est question de deux opinions (« opinion contraire à l'opinion commune $»$ ) ; la rhétorique parle de phrases antithétiques (et l'antithèse est définie comme "opposition de deux pensées, de deux expressions que l'on rapproche dans le discours pour en faire mieux ressortir le contraste ", Petit Robert 1979) ou identifie le paradoxe à l'oxymore («związek frazeologiczny obejmujący $d w a$ opozycyjne znaczeniowo wyrazy», Stownik terminów literackich 2000 ; « figure qui consiste à allier deux mots de sens incompatibles ", Petit Robert 1979) (cf. aussi Landheer 1996, qui définit le paradoxe comme fondé sur le mécanisme «à la fois X et non-X $\gg)$.

Le paradoxe de langue résulte donc d'une union discursive de deux éléments incompatibles, opposés, voire contradictoires l'un par rapport à l'autre relativement à une norme socialement codifiée dans la langue ou résultant du contexte verbal. 
Mais quels sont ces éléments? S'agit-il de deux énoncés, de deux mots, de deux éléments supérieurs à l'énoncé ( $p$. ex. enchaînements discursifs) ou inférieurs au mot (sèmes)? L'explication de leur nature relève déjà de la linguistique, et plus spécifiquement de la sémantique, ce qui n'est plus la question de cet article. C'est pourquoi je ne ferai qu'esquisser ici les principales possibilités de la recherche sur le paradoxe au sein de ce domaine ; la définition générale formulée ci-dessus semble leur être commune : c'est au niveau des détails que les divergences apparaissent.

\section{CONCLUSION :}

\section{DIRECTIONS DE LA RECHERCHE LINGUISTIQUE SUR LE PARADOXE}

Objet de recherche transdisciplinaire, le paradoxe est défini d'une façon plus ou moins restreinte dans le cadre de la logique, de la philosophie ou de la rhétorique. Ces perspectives-là s'entremêlent dans les entrées lexicographiques dont j'ai essayé de proposer une synthèse pour en arriver à une définition "prototypique " du paradoxe (l'accent mis sur le paradoxe verbalisé, c'est-à-dire le paradoxe de langue). Celui-ci, répétons-le, peut être considéré d'une façon générale comme relation de jonction établie sur l'axe syntagmatique du discours entre deux éléments incompatibles, opposés, voire contradictoires entre eux relativement à une norme socialement codifiée dans la langue ou résultant du contexte verbal.

Le phénomène semble extrêmement intéressant en ce qui concerne son mécanisme sémantique, ainsi que ses effets discursifs. Il est donc étonnant, comme le souligne B. Godart-Wendling (1996), que la linguistique ait si longtemps montré un désintérêt quasi total au problème du paradoxe : les recherches systématiques sur ce dernier dans la perspective strictement linguistique datent pour la plupart des années 1990 !

Parmi les principales théories permettant de traiter le problème du paradoxe, il convient d'énumérer :

1) La sémantique argumentative de Ducrot et de ses collaborateurs (théorie de l'argumentation dans la langue).

Le paradoxe, en tant que problème majeur posé à l'ADL, a été d'abord considéré dans le cadre de la théorie des topoï (cf. Bruxelles, Ducrot et Raccah 1995) et par rapport à la conception de la polyphonie (Ducrot 1989) ${ }^{9}$. L'énoncé paradoxal

9 Il convient de souligner que c'est une conception dont le succès est remarquable dans la recherche sur le paradoxe. Bien que ses auteurs eux-mêmes l'aient considérée comme insuffisante, différents chercheurs (p. ex. Tuţescu 1996, Landheer 1996) s'y réfèrent souvent pour expliquer le mécanisme du paradoxe. En ce qui concernc le mécanisme de la polyphonie (deux ou plusieurs « voix » coexistant dans un même ćnoncé), il est à associer au problème des univers de croyance (Univers $\mathrm{s}_{1}$ du locuteur ne coïncide le plus souvent pas avec l'Univers ${ }_{2}$ de son destinataire ou d'un autre utilisatcur de 
y est défini comme combinant les voix d'au moins deux énonciateurs, celle de l'énonciateur dit lexical, qui pose les topoï inclus dans la signification des mots, et celle d'un autre énonciateur, qui affirme le contraire exprimé par ces topoï et auquel s'identifie le locuteur.

Cette conception du paradoxe a été reformulée - et restreinte - dans la théorie des blocs sémantiques (cf. Carel et Ducrot 1999a et 1999b). Dans cette perspective, la plus récente, le paradoxe est défini en fonction du comportement argumentatif des mots ou des énoncés qui le créent ; ainsi, « un mot [ou énoncé] est linguistiquement paradoxal (LP) lorsque son argumentation inteme comporte des enchaînements linguistiquement paradoxaux " (Carel et Ducrot 1999a : 23). Quant à l'enchaînement, il est linguistiquement paradoxal si, dans le mot ou l'énoncé considéré, $a$ DONC $b$ n'est pas linguistiquement doxal ${ }^{10}$, tandis que a POURTANT $b$ est linguistiquement doxal (ex. : Paul est imprudent DONC Marie lui fait confiance / Paul est imprudent POURTANT Marie lui fait confiance) et vice versa (ex. : Paul est prudent DONC il est arrivé indemne / Paul est prudent POURTANT il est arrivé indemne).

2) La sémantique des univers de croyance, élaborée par Robert Martin (1987, 1992) et appliquée au problème du paradoxe par Tuțescu (1996).

La sémantique des univers de croyance s'occupe de la vérité langagière qui, liée au caractère flou du langage humain, est bien différente de la vérité logique. Selon cette théorie, l'application des valeurs aléthiques et déontiques à un énoncé se fait dans un univers de croyance (U) de l'énonciateur. Cet univers peut comporter deux sortes de mondes possibles : mondes potentiels et mondes contrefactuels (qui, respectivement, comportent ou ne comportent pas de propositions contradictoires avec le monde que l'énonciateur considère comme réel). Quant au paradoxe, il "convoque (...) deux univers de croyance : l'un, $\mathrm{U}_{1}$, potentiel, réel ou véritatif; l'autre, $\mathrm{U}_{2}$, contrefactuel, irréel » (Tuțescu 1996:80), il constitue ainsi une rencontre discursive de deux mondes possibles qui s'excluent mutuellement.

3) La sémantique textuelle (discursive) qui étudie les textes dans leur totalité et en rapport avec l'intertexte, en mettant l'accent sur la dimension sociale de la langue.

Dans cette perspective, la principale référence concernant le paradoxe est la recherche de Landheer $(1992,1996)$ qui classe les paradoxes selon le critère textuel (paradoxes intra-, inter- et extratextuels), rend compte de différents mécanismes et types de ce phénomène (paradoxe antonymique : $\mathrm{X}$ et le contraire de $\mathrm{X}$; paradoxe antanaclastique : $\mathrm{X}$ et non-X ; paradoxe synonymique : $\mathrm{X}$ et non-\{synonyme de $\mathrm{X}\}$; paradoxe hyponymique : $\mathrm{X}$ et non-\{hyperonyme de $\mathrm{X}\}$, cf. $1996: 112)$ et, surtout,

\footnotetext{
la langue, cf. Martin 1987. Tulescu 1996) et avec ce que Rastier (1987) décrit sous le nom de dissimilation discursive des contenus sémantiques (il s'agit plus particulièrement de la dissimilation d'univers).

${ }^{10} \mathrm{C}$ 'est-à-dire l'aspect auquel il appartient ne $\mathrm{s}^{\prime}$ inscrit pas dans la signification intrinsèque de ce mot ou énoncé.
} 
met l'accent sur son comportement discursif. A travers l'analyse de l'évaluation véhiculée par un énoncé paradoxal et des problèmes liés à la dénotation et à la connotation, Landheer souligne la spécificité du mécanisme du paradoxe («mécanisme de bascule ») qui ne s'actualise que pour se résorber aussitôt dans le contexte discursif.

Il importe de citer aussi la conception de Rastier (1996) qui privilégie la dimension sociale et idiolectale du texte (spécificité du milieu et de l'époque où vit l'auteur des séquences paradoxales", sa culture, ses convictions, etc.). Rastier envisage le paradoxe par rapport aux « seuils d'acceptabilité », concept utilisé souvent dans les recherches lexicologiques et qui sert ici à rendre compte de la hiérarchie des domaines sémantiques valorisés par le sens commun. En effet, dans une séquence paradoxale, cette hiérarchie se trouve renversée et les seuils d'acceptabilité déplacés, ce qui favorise des parcours interprétatifs «paradoxaux ».

D'une façon générale, la définition "prototypique » du paradoxe que j'ai proposée ci-dessus semble être valide pour toutes les théories signalées : il s'agit là toujours d'une opposition binaire ${ }^{12}$ qui, codifiée dans la langue ou actualisée dans le discours, surprend le destinataire par le fait que ses éléments se trouvent rapprochés dans une même séquence allant ainsi à l'encontre de ce qu'on considère comme la doxa, « opinion communément admise ».

\section{LISTE DES OUVRAGES CITÉS}

\section{Dictionnaires et encyclopédies}

Beauzee N., Marmontel J.-F. (1982, 1784, 1786). Encyclopédie méthodique. Grammaire \& Littérature, 3 tomes, Panckoucke et Plomteux, Paris ct Liège.

Dictionnaire étymologique, (1964), Larousse, Paris.

Didcrot D., D'A lembert J.L. (1969), Encyclopédie ou Dictionnaire raisonné des sciences, des arts et des métieurs, par une société de gens de lettres, 20 vol., Briasson, David, le Breton, Paris. «1751-1765».

Glowiński M., Kostkiewiczowa T., Okopień-Slawińska A., Slawiński J. (2000), Slownik terminów literackich, Ossolineum. Wrocław.

Grand Larousse de la langue française (1976), Paris.

Jedynak S. (red.) (1996), Mala encyklopedia filozofii, Oficyna Wydawnicza Branta. Bydgoszcz.

Julia D. (1964), Dictionnaire de la philosophie, Larousse, Paris.

Komputerowy slownik jezyka polskiego (1997), Wydawnictwo Naukowe PWN, Warszawa.

"Rastier analyse le paradoxe dans l'ceuvre de Chamfort (XVIII ${ }^{\mathrm{e}}$ siècle).

${ }^{12}$ Entre deux valeurs argumentatives de l'énoncé, deux univers de croyance, deux antonymes, deux acceptions du même mot, deux synonymes, un hypcronyme et un hyponyme, deux hiérarchies évaluatives des domaines sémantiques (sociale et individuelle), etc. 
Kopaliński W. (1967), Slownik wyrazów obcych i zwrotów obcojęzycznych, Wiedza Powszechna, Warszawa.

Le Petit Robert (1979), Société du Nouveau Littré, Paris.

Morier H. (1961), Dictionnaire de poétique et de rhétorique, PUF, Paris.

Slownik wyrazów obcych (1964), Państwowy Instytut Wydawniczy, Warszawa.

\section{Théorie}

A ristote (1994), Organon, (I. Catégories, II. De l'interprétation), J. Vrin (éd.), Paris (cité par D. Bertrand : 2000).

Bertrand D. (2000), Précis de sémiotique littéraire, Nathan, Paris.

Bruxelles S., Ducrot O., Raccah P.-Y. (1995), Argumentation and the lexical topical fields, Journal of Pragmatics, vol. 24, pp. 99-114.

Carel M., Duc rot O. (1999a), Le problème du paradoxe dans une sémantique argumentative, Langue française, $\pi^{\circ} 123$, pp. 6-26.

Carel M., Ducrot O. (1999b), Les propriétés linguistiques du paradoxe : paradoxe et négation, Langue française, $n^{\circ} 123, \mathrm{pp} .27-40$.

Douay-Soublin F. (1996), Le Paradoxe et son cortège : de l'" Encyclopédie " à l" Encyclopédie méthodique ", [in] R. Landheer, P.J. Smith, Le paradoxe en linguistique et en littérature, coll. "Histoire d'idées et critique littéraire", vol. 350, Droz, Genève, pp. 221-237.

$\mathrm{Dubois} \mathrm{J}$. et $\mathrm{Cl}$. (1971), Introduction à la lexicographie : le dictionnaire, coll. "Langue et Langage", Larousse, Paris.

Ducrot O. (1989), Topoï et sens, Actes du $9^{\text {eme }}$ colloque d'Albi, Université de Toulouse-Le Mirail, pp. 1-22.

Fontanier P. (1968), Les figures du discours, Flammarion, Paris.

Godart-Wendling B. (1996), "Je mens »: Histoires sémantique et logique d'un paradoxe, [in] Landheer R., Smith P.J., Le paradoxe en linguistique et en littérature, coll. " Histoire d'idées et critique littéraire "', vol. 350, Droz, Genève, pp. 17-38.

Greimas A. J. (1966), Sémantique structurale, Larousse, Paris.

Kripke S. (1975), Oulline of a Theory of Truth. The Journal of Philosophy, $n^{\circ} 72$, pp. 690-716 (cité par B. Godart-Wendling 1996).

Landheer R. (1992), Le paradoxe ou la tension d'une contradiction apparente, [in] M. Balat et J. Deledalle-Rhodes (éds), Signs of Humanity. L'homme et ses signes, Mouton de Gruyter, Berlin, vol. I, pp. 473-480.

Landhcer R. (1996), Le paradoxe: un mécanisme de bascule, [in] R. Landheer, P.J. Smith, Le paradoxe en linguistique et en littérature, coll. « Histoire d'idées et critique littéraire", vol. 350 , Droz, Genève, pp. 91-116.

Martin R.L. (1967), Toward a Solution to the Liar Paradox. The Philosophical Rewiew, $\mathrm{n}^{\circ} 76$, pp. 279-311 (cité par B. Godart-Wendling 1996).

Martin R. (1987), Langage et croyance. Les " univers de croyance " dans la théorie sémantique, Pierre Mardaga, Bruxelles.

Martin R. (1992), Pour une logique du sens, PUF, Paris.

Molini e G. (1992), Dictionnaire de rhétorique, Les Usuels de Poche, Paris.

Rastier F. (1987), Sémantique interprétative, PUF, Paris. 
Rastier F. (1996), Chamfort : le sens du paradoxe, [in] R. Landheer, P.J. Smith, Le paradoxe en linguistique et en littérature, coll. "Histoire d'idées et critique littéraire", vol. 350, Droz. Genève, pp.119-143.

Riffaterre M. (1996), Paradoxe et présupposition, [in] R. Landheer, P.J. Smith, Le paradoxe en linguistique et en littérature, coll. "Histoire d'idées et critique littéraire », vol. 350, Droz, Genève, $p$ p. 149-171.

Russell B., Whitehead A.N. (1910/1927), Principia mathematica, 3 vol., Cambridge University Press, Cambridge (cité par B. Godart-Wendling 1996).

Tarski A. (1972), Le concept de vérité dans les langages formalisés, [in] Logique. Sémantique, Métamathématiques, articles de 1923 à 1944, Colin. Paris, 1. I., pp. 157-269 (cité par B. Godart-Wendling 1996).

Tutescu M., Paradoxe, univers de croyance et pertinence argumentative, [in] R. Landheer, P.J. Smith, Le paradoxe en linguistique et en littérature, coll. " Histoire d'idées et critiquc littéraire », vol. 350, Droz, Genève, pp. 75-90.

Van der Poel M. (1996), "Paradoxon" et "adoxon" chez Ménandre le Rhéteur et chez les humanistes du XVF siècle, [in] R. Landheer, P.J. Smith, Le paradoxe en linguistique et en littérature, coll. "Histoire d'idées et critique littéraire », vol. 350, Droz, Genève, pp. 199-220. 